\title{
Statistical Properties of Inter-arrival Times Distribution in Social Tagging Systems
}

\author{
Andrea Capocci \\ University "La Sapienza" of \\ Rome, Italy \\ P.le Aldo Moro, 5, Rome, Italy \\ Vito D.P. Servedio \\ University "La Sapienza" of \\ Rome, Italy \\ P.le Aldo Moro, 5, Rome, Italy
}

\author{
Andrea Baldassarri \\ University "La Sapienza" of \\ Rome, Italy \\ P.le Aldo Moro, 5, Rome, Italy \\ Vittorio Loreto \\ University "La Sapienza" of \\ Rome, Italy \\ P.le Aldo Moro, 5, Rome, Italy \\ ISI Foundation \\ Villa Gualino, Turin, Italy
}

\begin{abstract}
Folksonomies provide a rich source of data to study social patterns taking place on the World Wide Web. Here we study the temporal patterns of users' tagging activity. We show that the statistical properties of inter-arrival times between subsequent tagging events cannot be explained without taking into account correlation in users' behaviors. This shows that social interaction in collaborative tagging communities shapes the evolution of folksonomies. A consensus formation process involving the usage of a small number of tags for a given resources is observed through a numerical and analytical analysis of some well-known folksonomy datasets.
\end{abstract}

\section{Categories and Subject Descriptors}

H.3.4 [Information Systems]: Systems and Software; H.3.1 [Information Storage and Retrieval]: Content Analysis and Indexing; G.2.2 [Mathematics of Computing]: Graph Theory

\section{General Terms}

Measurement, Theory

\section{Keywords}

folksonomies, semiotics, semiotic dynamics, small worlds

\section{INTRODUCTION}

The science of online social networks has recently become a interdisciplinary research field, since the technological environment and the number of interacting agents requires

Permission to make digital or hard copies of all or part of this work for personal or classroom use is granted without fee provided that copies are not made or distributed for profit or commercial advantage and that copies bear this notice and the full citation on the first page. To copy otherwise, to republish, to post on servers or to redistribute to lists, requires prior specific permission and/or a fee.

HT'09, June 29-July 1, 2009, Torino, Italy.

Copyright 2009 ACM 978-1-60558-486-7/09/06 ...\$10.00. the contribution of researchers such as computer scientists, physicists and sociologists. A particular example of such social systems are folksonomies $12,7,17,16$, i.e. online communities of users who, interacting through the World Wide Web, collaboratively build large and public knowledge bases of discrete resources such as bookmarks, scientific papers and digital images. Moreover, folksonomy users participate also in the classification of individual resources, by labeling each of them with arbitrarily chosen tags, that is, a (typically small) number of keywords describing each resource.

Folksonomies act both as public sources of information and as a storage system for single users, who selfishly collect resources for their own private use. These two tasks may push the evolution of these systems in opposite directions 13. As regards the first purpose, the development of cooperative behavior among users is crucial. Users have to agree on tag semantics, so that the tag-based classification of resources be coherent and readable. But, on the other hand, the popularity of such communities depends on the small effort demanded to users in the addition of elementary information units, whose description by tags, though simple, is very approximated 18 . Besides, the cultural background, the effort and the needs of users vary a lot throughout the community. This often generates ambiguous, incomplete or incoherent descriptions of the collected information and affects the whole accessibility of it.

A fundamental mechanism of consensus building among users is imitation. For example, consensus triggers the adoption of a given tag by many users when describing a resource or a whole set of resources, for descriptive or even strategic purposes. So, the social patterns of users' interaction reflect onto the statistical distribution of tags' usage. A highly skewed distribution in the usage of tags has already been observed, showing that their occurrences vary over many magnitudes [4, 8, 15. This reminds the Zipf law observed in written texts 19], where the occurrence of words is distributed according to a power-law. So, the skewness of the tag frequency distribution may be generated by endogenous mechanisms, or alternatively be the results of the statistical properties of the underlying language.

Less attention has been devoted so far to the statistics of tag dynamics. The growth of the vocabulary, i.e. the 
number of distinct tags as a function of time, has been empirically discovered to be sub-linear in different social tagging systems, and appropriate models have been developed to reproduce such growth rate, along with the frequency distribution of tags 4,9 .

Frequent and rare tags, of course, occur with different inter-arrival times, but a clear picture of correlations of the same tags by different users has not been drawn so far. In the following, we will study the statistics of inter-arrival times in some well-known collaborative tagging system, where the large number of users allows a reliable empirical analysis, and will try to find evidences in favor or against the presence of correlation and collaboration patterns through the detection of regularities in the temporal statistics of tags arrival.

Similar analysis have already been performed for other data sets, namely texts, showing that the distribution of word occurrence is not random and deviation from a Poissonian picture are present. Fat tails in the distribution of word inter-arrival times have been detected in texts, and put into relation with the underlying semantics 6, 11, 14, 2 , 1. We will focus here on a different kind of word sequence, that is, the sequence of tags used by annotating users in some web-based social tagging community, to describe the relative resources.

\section{DATASETS}

The datasets studied here describe the tagging activities in some well-known collaborative bookmarking websites including del.icio.us, Bibsonomy and CiteULike. The data reports individual tag assignments posted by users in chronological order. Each tag assignment is a triplet formed by a user, a resource and a tag. Resources are URL in del.icio.us, while Bibsonomy and CiteULike collect scientific citations. Tags are keywords associated by users to describe resources. Each user can assign an arbitrary number of tags to the same resource in a single post, so more than one tag assignments may come at the same time.

Such datasets comprises 140306315 tag assignments, with 2482873 tags for 18778597 resources for the bookmarking website del.icio.us. The The CiteULike dataset collects 571340 tag assignments with 199512 resources and 51080 tags. The Bibsonomy dataset includes 671808 tag assignments, with 206942 resources and 58756 tags.

\section{TAG DYNAMICS: OBSERVATIONS}

Correlations in the behavior of user collaborating in tagging resources online can be studied by inspecting the temporal statistics of tag usage. Time, here, is discrete e is measured in number of successive posts. For example, one can study the inter-arrival time of tags, that is, the time length occurring between two subsequent tag assignments involving the same tag. If users behave independently, tags are added with a constant probability at each time unit. Accordingly, the arrival of tag would be described by a Poissonian process, where each occurrence is uncorrelated from the previous one. In this case, inter-arrival times are distributed according to an exponential distribution with a well-defined average inter-arrival times given by $1 / f$, where $f$ is the tag frequency [5].

By contrast, observed individual tag inter-arrival times distribution shows that inter-arrival times span over all time

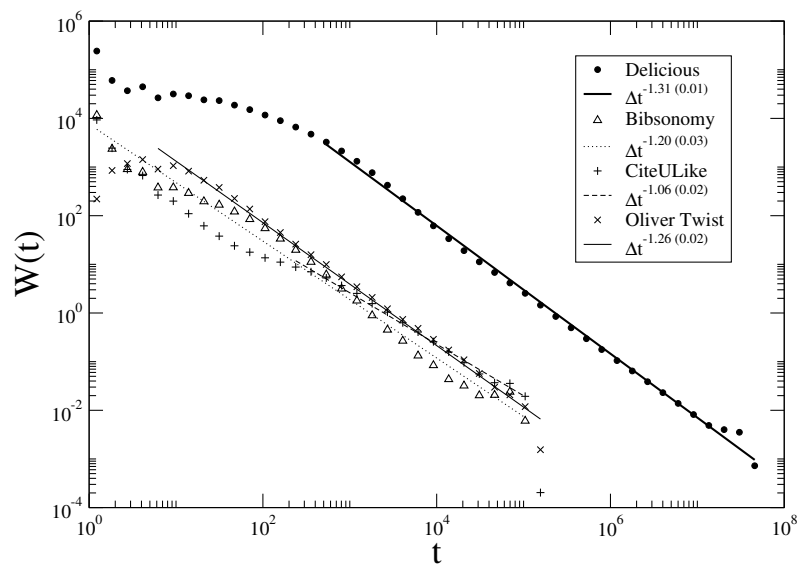

Figure 1: Tag inter-arrival times distribution $W(t)$ in collaborative tagging communities as a function of $t$

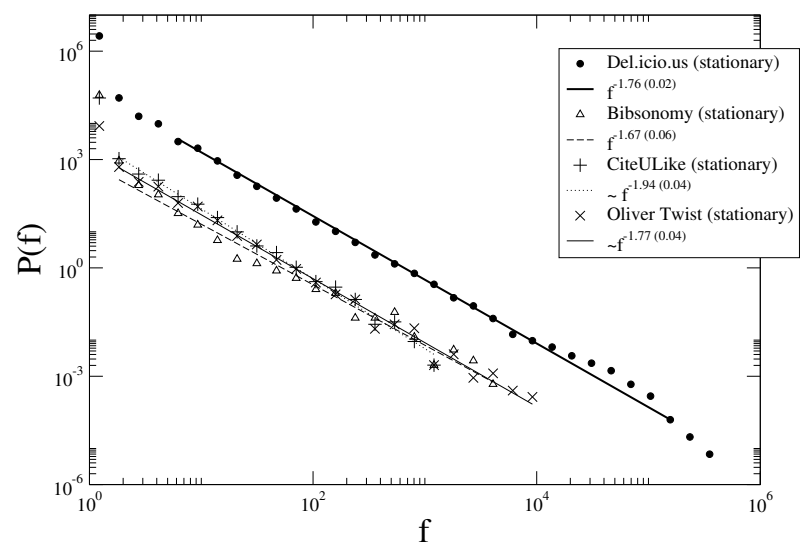

Figure 2: Stationary tag occurrence distribution in collaborative tagging communities.

scales, with a fat-tailed distribution, as shown in figure 1 The number of inter-arrival times of time length $t$, computed over all tags, is a power law $W(t) \propto t^{-\gamma}$, with $\gamma \simeq 1.3$ in different tagging systems.

The latter analysis has been performed on a subset of "stationary" tags, that is, tags that occurs throughout the whole datasets. This aims to exclude tags that start or stop occurring in the dataset during the time window covered by it. These could be frequently occurring tag with short typical inter-arrival time, though their observed frequency maybe small because of the partial overlap between the dataset time window and their lifespan. Thus, a tag with frequency $f$ is called "stationary" if its first occurrence time and the time interval between its last occurrence and the end of the dataset time window are both lesser than $1 / f$.

Nevertheless, this power-law behavior maybe the consequence of the uneven distribution of tag occurrences, which is known to follow a Zipf law. As reported in figure 2, the number of tags occurring $f$ times is a power-law $P(f) \propto f^{-\beta}$ in several collaborative tagging communities, with $\beta \simeq 1.7$.

The fat tails in figure 1 may be determined by the large number of tags with low frequency (i.e., long inter-arrival times). To verify this, one reshuffles the time ordering of 


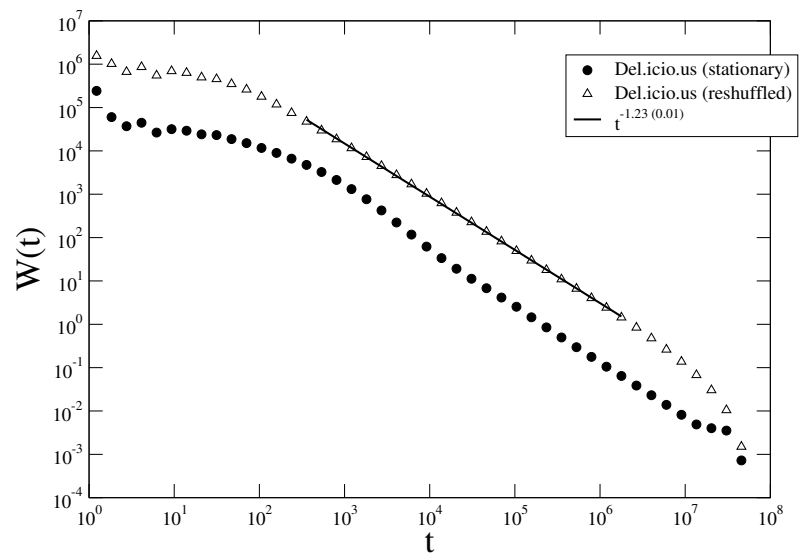

Figure 3: Comparison between the tag inter-arrival times distribution in the original Del.icio.us dataset (stationary tags) and in an artificial one where the time-ordering of tag assignments has been randomly reshuffled.

tags by reassigning them to randomly chosen posts. This way, time correlations are removed and the distribution of inter-times is determined solely by the Zipf law in the frequency of tags. From now on, we limit our statistical analysis to the larger Del.icio.us dataset, where richer data allow a more reliable statistical analysis. However, the numerical and analytical results presented here hold within a reasonable approximation in other social bookmarking communities. As checked in figure 3 the inter-arrival time distribution changes slightly from the power-law behavior described above. Therefore, the distribution $W(t)$ is no signature of complex correlation patterns. This can also be easily understood by a simple analytical argument, shown in the next section.

Thus, one should observe individual tag inter-arrival time distribution. which, of course, display a poorer statistics. Here one finds different patterns for high-frequency tags and low-frequency tags. The first display a fast decay in the distribution for large values of the inter-arrival time. Reasonably, tags that occur less frequently display longer interarrival times with a finite probability. Their inter-arrival times distribution decay as a power law for large values of $\Delta t$.

The presence of power laws in the distribution of interarrival times is often put in strict relation with processes taking place in "avalanches", i.e. with long period of stability with sudden bursts of activity of all scales of magnitudes, limited only by finite size effects, as shown in an example reported in figure 5. Scale-invariance in the distribution of inter-time distribution corresponds to unpredictability of future events, given the past time series 10 .

By reshuffling tags, time correlations would be removed, and the curves corresponding to those plotted in figure 3 would exhibit an exponential decay

\section{INTER-ARRIVAL TIMES DISTRIBUTION}

The statistics of inter-arrival times for high-frequency and low-frequency tags can be simply related. The inter-arrival time distributions of individual tags can be modeled by a scaling function depending on the tag frequency $f$, written

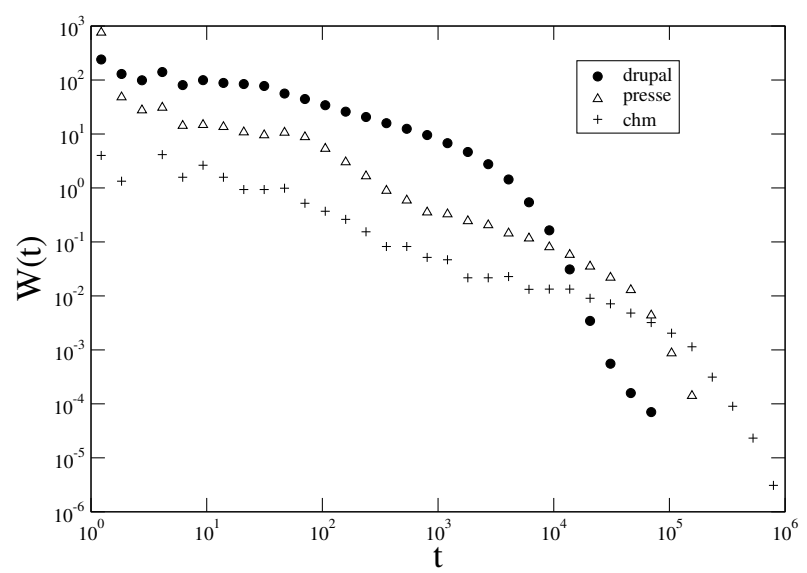

Figure 4: The inter-arrival times distribution for individual tags "drupal" (33442 occurrences), "presse" (5011) and "chm" (999) in the collaborative tagging system del.icio.us.

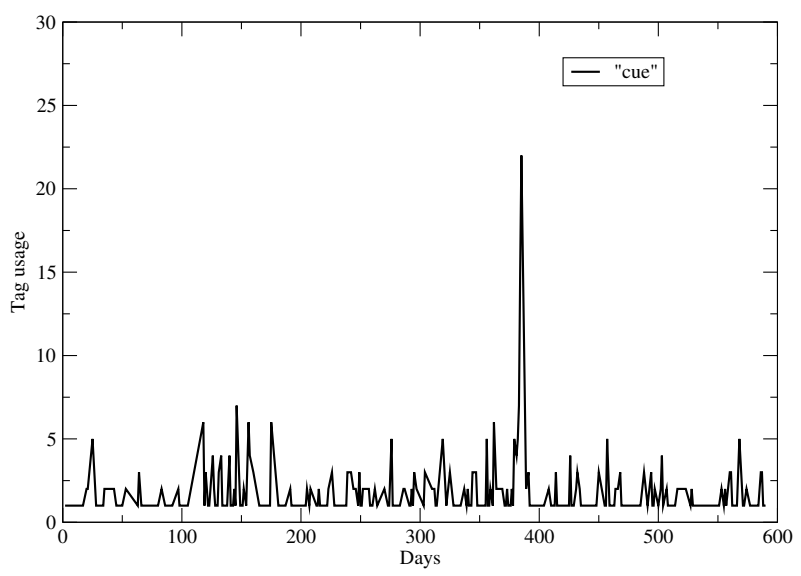

Figure 5: The weekly usage of the tag "cue" during the two years covered by the del.icio.us dataset, displaying periods of high activity and sharp activity peaks. The $\mathrm{x}$-axis reports the number of weeks since 1st January 1970 


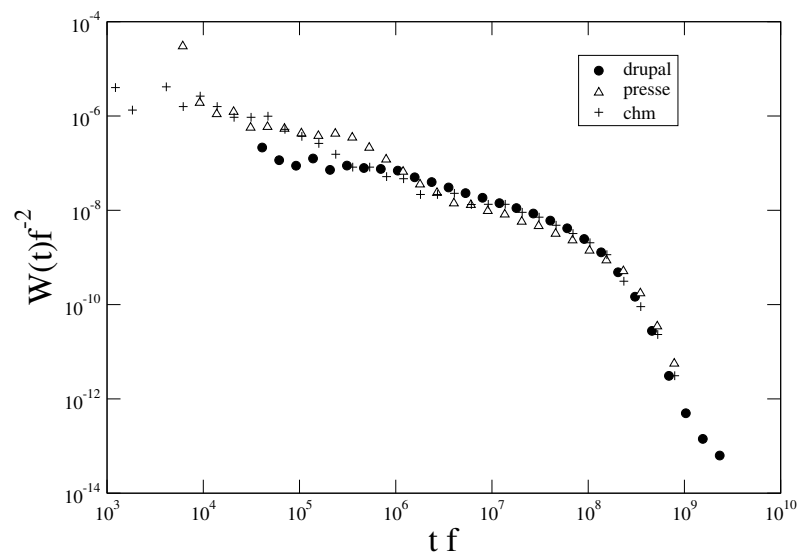

Figure 6: The cutoff function $g(f t)=\frac{W_{f}(t)}{f^{2-\alpha} t^{-\alpha}}$ plotted against $f t$ for the inter-arrival times distribution of individual tags "drupal" (33442 occurrences), "presse" (5011) and "chm" (999) in the collaborative tagging system del.icio.us.

as

$$
W_{f}(t) \propto R(f) t^{-\alpha} g\left(f^{\psi} t\right)
$$

where $W_{f}(t)$ is the number of inter-arrival times of time length $t$ for a tag of frequency $f+1$, and $g$ is a function which is constant for low values of the argument and decays rapidly after a given cut-off value, i.e. $g(x)=1$ for $x \ll 1$ and $g(x)=0$ for $x \gg 1$. Since the total number of intertimes for a tag with frequency $f+1$ is $f, W_{f}(t)$ is normalized by

$$
\int_{0}^{\infty} W_{f}(t) d t=f
$$

By using the definition 1 this leads to

$$
f \propto R(f) f^{\psi(\alpha-1)} \int_{0}^{\infty} x^{-\alpha} g(x) d x
$$

where $x=f^{\psi} t$, so that $R(f)=f^{1+\psi(1-\alpha)}$.

The inter-arrival times distribution observed over all tags $W(t) \propto t^{-\gamma}$, which receives contribution by the occurrence of tags of all frequencies distributed according to the law 2 . can be written as

$$
W(t)=\int_{0}^{\infty} P(f) W_{f}(t) d f
$$

which, after replacing $P$ and $W_{f}$ by their functional form, reads

$$
W(t) \propto t^{\frac{\beta-2}{\psi}-1} \int_{0}^{\infty} x^{\beta(1-\alpha)-1+\frac{1}{\psi}} g(x) d x .
$$

. Thus, one obtains the relation $\psi=\frac{2-\beta}{\gamma-1}$. By replacing the observed values for $\beta$ and $\gamma$, the relation yields $\psi \simeq 1$ for del.icio.us, $W(t) \propto t^{\beta-3}$ and

$$
W_{f}(t) \propto f^{2-\alpha} t^{-\alpha} g(t f)
$$

. The value of the exponent $\alpha \simeq 0.75$, measured by the inter-arrival times distribution in del.icio.us, is verified in the figure 6. where the inter-arrival times distributions for tags with different frequencies collapse on the same function.

After reshuffling tag order as described above, since the arrival of tags is now a Poissonian process, the distribution

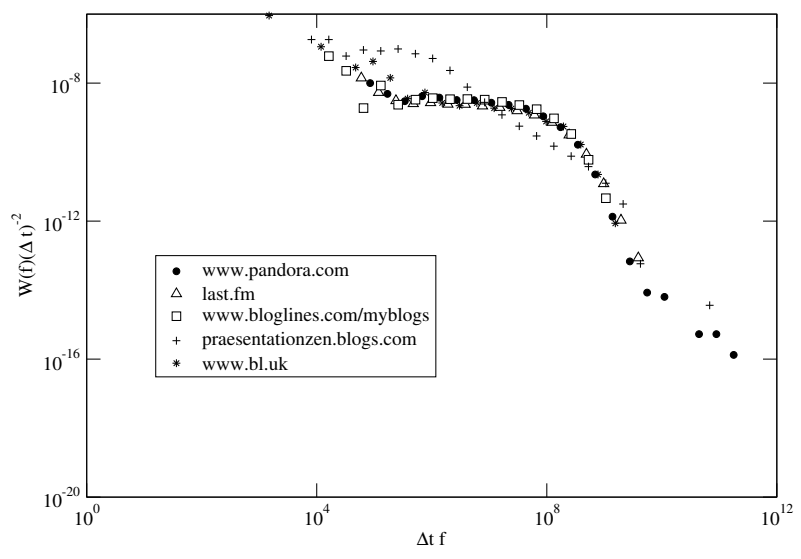

Figure 7: The distribution of inter-arrival times between subsequent tag assignments involving the same resource.

6 changes into an exponential function, with inter-arrival times statistics equal to $W_{f}^{(P)}(t) \propto f \lambda(f) e^{\lambda(f) t} . \quad \lambda(f)$ is the average inter-arrival times, equal to $f / T$ where $T$ is the time length of the observed period. The distribution $W(t)$ can thus be computed as

$$
W(t)=\int_{0}^{\infty} P(f) f \lambda(f) e^{-\lambda(f) t} d f
$$

that, by replacing the power-law form of $P(f)$ yields $W(t) \propto$ $t^{\beta-3}$, showing that the reshuffling changes only the singletag inter-arrival times distribution but leaves unchanged the overall inter-arrival times distribution. Therefore, the powerlaw behavior observed for $W(t)$ depends only on the frequency skewed distribution and cannot be used to study the dynamical properties of tag arrival.

\section{COLLABORATIVE PATTERNS}

The bursty behavior of tagging activities is not in itself a signature that complexity arises due to the interaction of users. A clearer sign of user cooperation can be found by analyzing the temporal pattern corresponding to individual resources. Inter-Arrival times $t$ between subsequent tagging of the same resource are distributed according to a power-law with a sharp cut-off for large values of $t$ going to infinity for less tagged resources, as displayed in figure 7. Since a user cannot tag a resource twice, the fact that individual resource are tagged in "avalanches" depends on the contribution of many users. By contrast, if users were tagging independently one from each other, $t$ should be distributed as an exponential random variable, as happens for Poissonian processes. The individual resource inter-arrival distribution can be analyzed as done above for tags, showing that resources are tagged in bursts spanning all time length scales.

A similar avalanche-like pattern can be observed by taking into account only the first usage of a tag by each user, and observing the inter-arrival times distribution $W_{f_{1}}\left(t_{1}\right)$ of this special tagging events, where $t_{1}$ refers to their time separation and $f_{1}$ is the number of such events. This way, one removes the possibility that the the short inter-arrival times are originated by users who often use a given tag for their own interests, and long inter-arrival times may come 


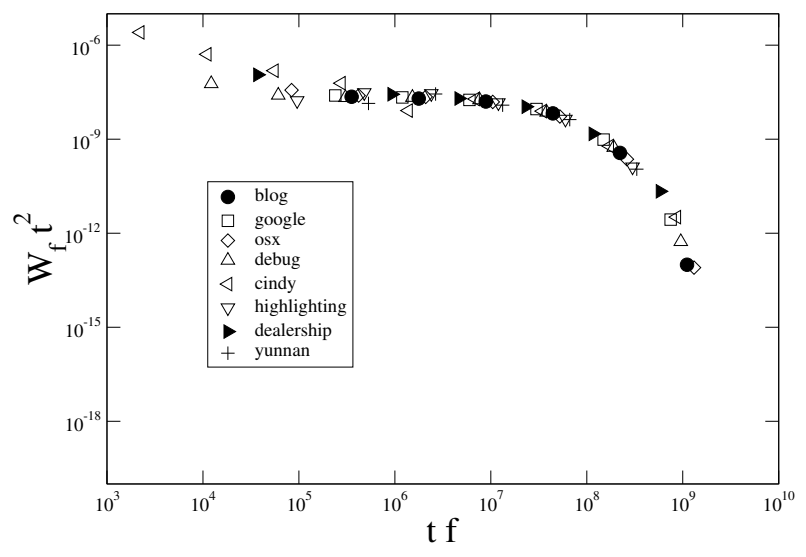

Figure 8: The distribution of inter-arrival times between the first usage of some tags by each user, divided by the number of such events $f_{1}$, plotted as a function of $t_{1} f_{1}$.

by the numerous users who seldom tag resources with that particular tag. If this was the case, the skewed distribution would just be the result of the superposition of heterogeneous, yet independent, usage patterns. Interestingly, the distribution of inter-arrival times of a given tag, when one limits the observation to the first usage of that tag by each user, follows the same statistics observed above when one takes into account the whole tagging activity. In particular, the relation reported in eq. 6holds also for the inter-arrival times $t_{1}$, as shows the collapse reported in figure 8 . If relation 6 holds, tags are "discovered" by users in a correlated and bursty manner.

However, this is not yet a proof of cooperation among online users. In fact, bursts of attention may arise by both a direct mutual influence between users one on each other; otherwise, users may independently be influenced by the same sources of information and news, where attention bursts may originate without any interaction among them.

The stream of tag assignment involving a given resource, though, carries a clearer evidence of users interaction. By plotting the number of distinct tags, i.e. the vocabulary, used for a resource as a function of the number of tag assignments to it, one observes a sub-linear vocabulary growth: so, the pace at which new tags are introduced by users to describe a resource decreases with time, and new tags are introduced less and lesser. In other words, users tend to employ the same tags used by previous peers when describing the same resources.

The figure 9 shows that the sub-linear relation between tag assignments and number of distinct tags involving a single resource holds for the large majority of them. Interestingly, this relation is not respected by "spam" bookmarks, that is, by tag assignments violating of the collective agreement about tag semantical organization. As other signatures of complex features, so, this relation may reveal useful in methods of spam detection [5, 3].

A deeper insight into the collective development of a tag vocabulary associated to a resource is provided by studying the Inverse Participation Ratio $(I P R)$ of tags in such vocabulary. Let $v_{i=1, \ldots, N}$ be the components of a vector $\mathbf{v}$, such that $\sum_{i=1, N} v_{i}^{2}=1$. The definition of $I P R$ is $I P R=$

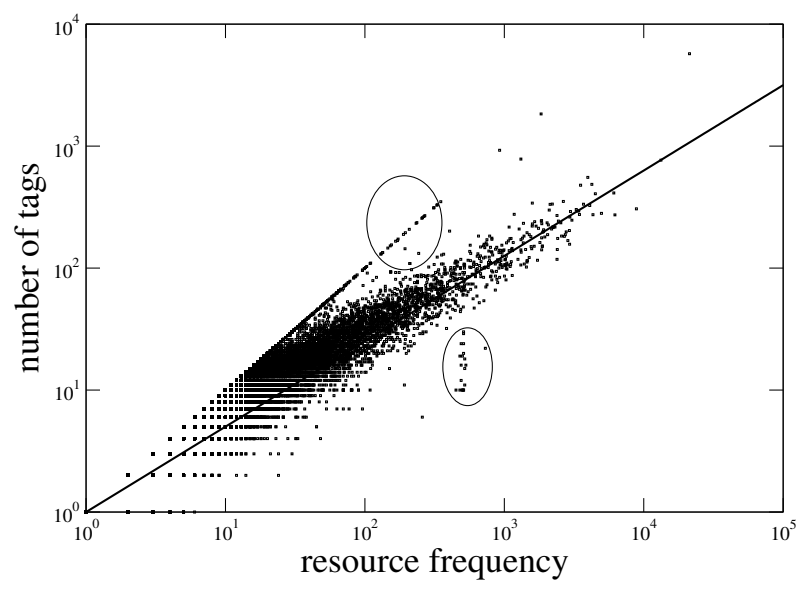

Figure 9: The number of distinct tags $n(f)$ assigned to a resource as a function of the number $f$ of tag assignments involving the resource. The majority of resources lie approximately on the line $n(f) \propto t^{0.7}$. The plot shows also two clusters of resources (in the ellipses) lying away from the line. A direct inspection reveal that that resources are malicious "spam" bookmarks.

$\sum_{i=1, N} v_{i}^{4}$. If all components are equal to $v_{i}=1 / \sqrt{N}, I P R$ is equal to $1 / N$. Conversely, if all components are null but one, $I P R=1$. So, the IPR describes the number of a vector components that contribute significantly to the vector norm. Analogously, the IPR of tag streams computed on the relative frequency of tags represents the number of significant tag used to describe a given resource.

As shown in figure 10 the number of significant tags is rather constant even for resources tagged thousands of times, showing that a consensus is reached among users about how to describe a given resource and following users do not add new significant tag but rather employ already used ones.

\section{CONCLUSIONS}

We have studied empirically and analytically the behavior of users in some well-known collaborative tagging online systems, where a large number of users collects resources and classify them by attaching a number of labels, called tags, to each of them. A resource can be tagged by many users, and thus be tagged by a large number of labels.

We have analyzed the statistics of inter-arrival times of tags, i.e. the time interval occurring between two subsequent occurrences of a same tag, and of resources. We have uncovered non-trivial statistical properties, which can be related to avalanches in the tagging activities. Such bursty behavior shows that the tagging activity by different users is strongly correlated. Regularities in the inter-arrival times distribution are studied analytically, so that the dynamics of rare and frequent tags can be unified by a unique law, which depends only on the frequency parameter $f$. Moreover, we have shown that users of tagging systems find a consensus about the tag description of each resource. In fact, we have empirically shown that the number of significant tags for each resource is rather constant, even for resources that have been tagged by thousand of heterogeneous users. A byproduct of our analysis regards the detection of spam in such 


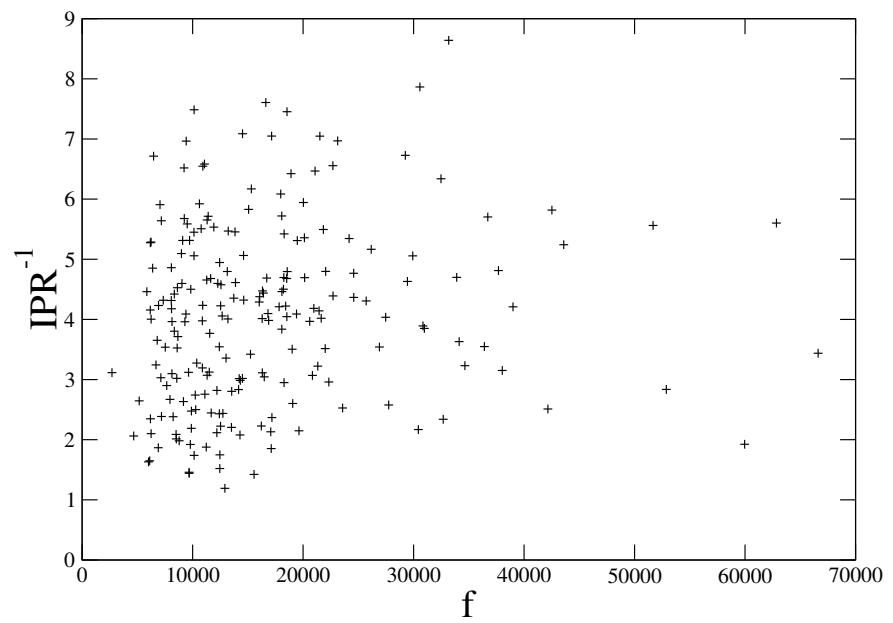

Figure 10: The $I P R$ of tag streams associated to individual resources as a function of the number of tag assignments $f$ involving them.

freely accessible communities. The number of distinct tags attached to a resource, i.e. the resource vocabulary length, grows sub-linearly with the number of tagging events involving that particular resource, with a relation which holds with good precision for a large majority of tags. Two well-defined subset of tags, however, do not satisfy such relationship between the resource occurrence and the resource vocabulary length. A direct inspection of such tags reveals that the latter have been added during malicious spam activity. This suggests a fast method to detect spam in collaborative tagging systems.

\section{ACKNOWLEDGMENTS}

This research was supported by the TAGora project (FP6IST5-34721) funded by the Future and Emerging Technologies program (IST-FET) of the European Commission. We thank C. Cattuto for many stimulating discussions.

\section{REFERENCES}

[1] E. Altmann, P. J.B., and M. A.E. Beyond word frequency: Bursts, lulls, and scaling in the temporal distributions of words, 2009.

[2] E. Alvarez-Lacalle, B. Dorow, J.-P. Eckmann, and E. Moses. Hierarchical structures induce long-range dynamical correlations in written texts. Proceedings of the National Academy of Science, 103:7956-7961, May 2006.

[3] A. Capocci and G. Caldarelli. Folksonomies and clustering in the collaborative system citeulike. Journal of Physics A: Mathematical and Theoretical, 41(22):224016+, 2008.

[4] C. Cattuto, V. Loreto, and L. Pietronero. Semiotic dynamics and collaborative tagging. Proceedings of the National Academy of Sciences, 104:1461, 2007.

[5] C. Cattuto, C. Schmitz, A. Baldassarri, V. D. P. Servedio, V. Loreto, A. Hotho, M. Grahl, and G. Stumme. Network properties of folksonomies. AI Communications, 20(4):245 - 262, 2007.

[6] K. W. Church and W. A. Gale. Poisson mixtures. Natural Language Engineering, 1:163-190, 1995.
[7] G. W. Furnas, C. Fake, L. von Ahn, J. Schachter, S. Golder, K. Fox, M. Davis, C. Marlow, and M. Naaman. Why do tagging systems work? In $\mathrm{CHI}$ '06: CHI '06 extended abstracts on Human factors in computing systems, pages 36-39, New York, NY, USA, 2006. ACM.

[8] S. A. Golder and B. A. Huberman. Usage patterns of collaborative tagging systems. J. Inf. Sci., 32(2):198-208, 2006.

[9] H. Halpin, V. Robu, and H. Shepherd. The complex dynamics of collaborative tagging. In $W W W$ '07: Proceedings of the 16th international conference on World Wide Web, pages 211-220, New York, NY, USA, 2007. ACM.

[10] J. H.J. Self-Organized Criticality. Cambridge University Press, Cambridge, UK, 1998.

[11] S. M. Katz. Distribution of content words and phrases in text and language modelling. Nat. Lang. Eng., 2(1):15-59, 1996.

[12] P. Mika. Ontologies Are Us: A Unified Model of Social $\mathrm{N}$ etworks and Semantics. In Y. Gil, E. Motta, V. R. Benjamins, and M. k A. Musen, editors, ISWC 2005, volume 3729 of $L N C S$, pages 522-536, Berlin Heidelberg, November 2005. Springer-Verlag.

[13] E. Rader and R. Wash. Tagging with del.icio.us: Social or selfish? In CSCW 2006 (poster), 2007.

[14] A. Sarkar, P. H. Garthwaite, and A. D. Roeck. A Bayesian mixture model for term re-occurrence and burstiness. In In: Proc. of the Ninth Conference on Computational Natural Language Learning (CoNLL-2005). Ann Arbor, Michigan. ACL. pp $48-55$, 2005.

[15] K. Shen and L. Wu. Folksonomy as a complex network, Sep 2005.

[16] H. Wu, M. Zubair, and K. Maly. Harvesting social knowledge from folksonomies. In HYPERTEXT '06: Proceedings of the seventeenth conference on Hypertext and hypermedia, pages 111-114, New York, NY, USA, 2006. ACM.

[17] X. Wu, L. Zhang, and Y. Yu. Exploring social annotations for the semantic web. In $W W W$ '06: Proceedings of the 15th international conference on World Wide Web, pages 417-426, New York, NY, USA, 2006. ACM.

[18] B. Yochai. Coase's penguin, or, linux and the nature of the firm. Yale Law Journal, 112, 2002.

[19] G. K. Zipf. Human Behavior and the Principle of Least Effort. Addison-Wesley (Reading MA), 1949. 